\title{
Genome Resources of Two Pathotypes of the Potato Cyst Nematode Globodera rostochiensis from New York
}

\author{
Xiaohong Wang, ${ }^{1,2, \dagger}$ Huijun Yang, ${ }^{1}$ Pierre-Yves Véronneau, ${ }^{3}$ David Thurston, ${ }^{2}$ and \\ Benjamin Mimee ${ }^{3, \dagger}$ \\ ${ }^{1}$ Robert W. Holley Center for Agriculture and Health, United States Department of Agriculture-Agricultural \\ Research Service, Ithaca, NY 14853, U.S.A. \\ ${ }^{2}$ School of Integrative Plant Science, Cornell University, Ithaca, NY 14853, U.S.A. \\ ${ }^{3}$ Agriculture and Agri-Food Canada, Saint-Jean-sur-Richelieu Research and Development Centre, \\ St-Jean-sur-Richelieu, Québec J3B 3E6, Canada
}

\begin{abstract}
The potato cyst nematode Globodera rostochiensis is a regulated pest posing a serious threat to potato production worldwide. Although the endemic pathotype (Ro1) of G. rostochiensis has been confined to New York State for several decades as a result of quarantine regulations and management with resistant potato cultivars, a virulent pathotype, Ro2, has emerged, for which control measures are scarce. The ability to detect Ro2 early in fields is necessary to sustain the success of $G$. rostochiensis quarantine in the United States. Here, we report the comparative analysis of whole-genome sequences of multiple single-cyst-derived Ro1 and Ro2 lines, propagated from original field populations. The identified discriminant variants are good targets for developing molecular diagnostic tools for differentiating $G$. rostochiensis pathotypes in New York.
\end{abstract}

Potato cyst nematodes (Globodera rostochiensis and G. pallida) are internationally recognized quarantine pests that cause tremendous negative impacts on potato production and trade globally (Dandurand et al. 2019; Evans and Stone 1977; Mai 1977). Strict quarantine regulations and deployment of potato cultivars containing the $\mathrm{H} 1$ resistance gene have successfully confined the endemic pathotype of $G$. rostochiensis Ro1 to very restricted areas in New York State since its original detection in the 1940s (Brodie and Mai 1989; Dandurand et al. 2019; Evans and Brodie 1980). However, a virulent pathotype that can reproduce well on $\mathrm{H1}$ containing potato cultivars has emerged and was characterized as Ro2 based on reproduction on the differential lines proposed by Kort et al. (1977) (Brodie 1996; Mimee et al. 2015; Whitworth et al. 2018). The current protocol for determining Ro2 relies on multiple rounds of bioassays to verify nematode multiplication on potato plants with the $\mathrm{H} 1$ gene, which is both labor intensive and time consuming. To ensure the continued success of $G$. rostochiensis quarantine in the United States, molecular tools for a rapid and reliable identification of G. rostochiensis pathotypes are urgently needed. Therefore, whole-genome sequencing of multiple single-cyst-derived Ro1 and Ro2 lines was performed to identify single-nucleotide polymorphisms and other sequence variations that may be used as diagnostic markers to rapidly differentiate $G$. rostochiensis pathotypes in New York.

${ }^{\dagger}$ Corresponding authors: X. Wang; xiaohong.wang@usda.gov; and

B. Mimee; benjamin.mimee@canada.ca

*The $e$-Xtra logo stands for "electronic extra" and indicates that supplementary tables are published online.

The author(s) declare no conflict of interest.

Accepted for publication 16 November 2020.
$e-X_{\text {tra* }}$

\section{Funding}

This study was supported by funding from United States Department of Agriculture (USDA) Agricultural Research Service and USDA National Institute for Food and Agriculture award number 2014-07639.

\section{Keywords}

genetic variation, Globodera rostochiensis, microbe-genome sequencing, nematode pathotype, nematodes, potato cyst nematode, whole-genome sequencing 


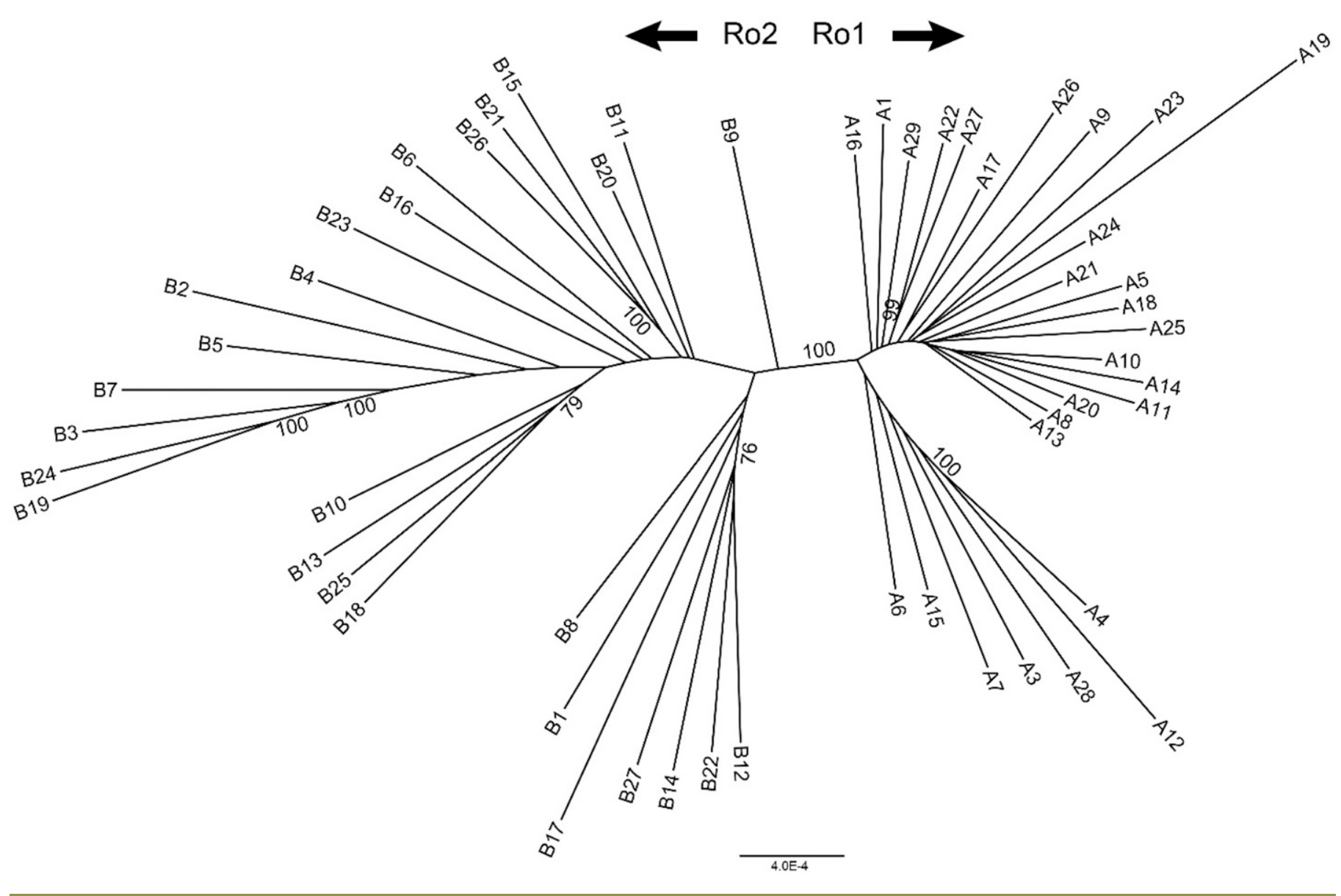

Fig. 1. Phylogenetic analysis of the 55 single-cyst lines of Globodera rostochiensis pathotypes Ro1 and Ro2 from New York, U.S.A. Neighbor-joining dendrogram in which samples "A" are Ro1 and "B" are Ro2. Bootstrap values $>70 \%$ are shown.

The Ro1 and Ro2 populations of G. rostochiensis used for generating single-cyst lines are lab-maintained populations, which represent an original collection of cyst samples from earlydetected fields, mainly in Steuben County, NY, and have since been propagated for generations on either an Ro1-susceptible plant (containing no $\mathrm{H} 1$ gene) (for the Ro1 population) or an Ro1resistant plant (containing the $\mathrm{H} 1$ gene) (for the Ro2 population) (Brodie 1996; Brodie and Mai 1989). Individual cysts from the Ro1 population or the Ro2 population were further multiplied for several generations on potato cultivar Katahdin (an Ro1-susceptible plant) (for the 28 Ro1 lines) or potato cultivar Pike (an Ro1-resistant plant) (for the 27 Ro2 lines) (Brodie 2003; Plaisted et al. 1998). Approximately 20 cysts from each of the single-cyst lines were used for genomic DNA extraction, as previously described (Mimee et al. 2015). TruSeq DNA library construction and Illumina NextSeq500 sequencing were performed at Cornell Institute of Biotechnology. Distinct libraries were prepared for each single-cyst line, barcoded, and pooled before sequencing (Supplementary Table S1). This yielded a total of $927,077,330$ paired reads.

Sequencing reads were trimmed with Trimmomatic v0.36 (Bolger et al. 2014) to a minimum length of $50 \mathrm{bp}$ and a minimum Phred quality score of 20 . Unpaired reads were discarded and the remaining sequences were aligned to the $G$. rostochiensis reference genome nGr.v1.1 (Eves-van den Akker et al. 2016) using BWA-MEM v0.7.12 (Li et al. 2009). Variants were identified using FreeBayes v1.2.0 (Garrison and Marth 2012) with minimum mapping quality set at 30 , minimum base quality at 20 , minimum alternate count at 2 , and minimum alternate fraction at 0.05 . In total, 2,909,165 variants were identified in the dataset. Additional filters were applied to keep only the variant calls with a minimum coverage of 5 in each of the 55 lines and no missing data. This resulted in a final list of 1,519,034 variants (supplemental vcf file at https:// datadryad.org/stash/dataset/doi:10.5061/dryad.rxwdbrv6b). A neighbor-joining phylogenetic tree including all of the lines was constructed using VCF-kit (Cook and Andersen 2017) with all of the variant sequences (Fig. 1). This analysis clearly separated the avirulent lines (Ro1) from the virulent lines (Ro2). 
Table 1. Summary statistics of the genome sequences of the 55 single-cyst lines of Globodera rostochiensis pathotypes Ro1 and Ro2 from New York, U.S.A.

\begin{tabular}{lc} 
Traits & Statistics \\
Number of Ro1 lines sequenced & 28 \\
Number of Ro2 lines sequenced & 27 \\
Mean number of reads per sample and coverage for & $16,491,553(25.8 \times)$ \\
Ro1 lines & \\
Mean number of reads per sample and coverage for & $16,809,573(26.3 \times)$ \\
Ro2 lines & \\
Overall coverage for Ro1 sequences & $722 \times$ \\
Overall coverage for Ro2 sequences & $710 \times$ \\
Number of variants identified & $1,519,034$ \\
Number of discriminant markers & 241 \\
\hline
\end{tabular}

a Number of sequencing reads per sample is detailed in Supplementary Table S1.

Variants that were discriminant for Ro1 versus Ro2 were identified by screening those that were fixed (homozygous) within either all of the Ro1 lines or all of the Ro2 lines and behaved differently in the other pathotype. Only the loci that had a single allele (homozygous) in all of the lines from a pathotype and a different allele in all of the lines from the other pathotype (either homozygous or heterozygous) were kept. Following this analysis, 241 loci that discriminate Ro1 lines from Ro2 lines were found (Supplementary Table S2). These variants were all homozygous in Ro2 (virulent), which is consistent with a reduced diversity at loci associated with strong directional selection. Interestingly, these loci were not randomly distributed across the genome but concentrated on only 18 scaffolds (out of 4,377), and 124 of 241 were located on a unique scaffold (GROS_00167) in a region spanning approximately $126 \mathrm{~kb}$. Statistics of genome sequencing and variants detection are summarized in Table 1.

The discriminant variants were annotated using SnpEff v4.3 (Cingolani et al. 2012) to predict their putative effect on gene and protein sequences. Of the 241 variants, 56 were located in predicted genes, of which 23 were in exons; these include a splice site variant with high predicted impact, 8 missense variants, and 14 low-impact mutations, mostly synonymous variants (Supplementary Table S2). All of the moderate or high-impact variants were found in only five genes ( $g 06866, g 06874, g 06875, g 11769$, and $g 11961)$ but none of them is a known effector gene. Although all of the 241 variants are good targets to develop a diagnostic tool to rapidly differentiate $G$. rostochiensis pathotypes in New York, those present in these five genes represent ideal markers because they could be functionally associated with the virulence phenotype. These genome sequences will be a critical resource to improve our ability to detect the Ro2 pathotype of $G$. rostochiensis and for future analyses on host-nematode interactions and the understanding of virulence in potato cyst nematodes.

Data availability. Whole-genome sequences of the Ro1 and Ro2 lines of G. rostochiensis were deposited at NCBI Sequence Read Archive in the BioProject PRJNA662608, with accession numbers SAMN16090804 to SAMN16090858. The supplemental vcf file containing the list of the 1,519,034 variants and information for each line is available at https://datadryad.org/ stash/dataset/doi:10.5061/dryad.rxwdbrv6b.

\section{Acknowledgments}

We thank the Genomics Facility of the Biotechnology Resource Center of Cornell Institute of Biotechnology for their help with sequencing experiments, and J. Lafond-Lapalme and E. Lord from Agriculture and Agri-Food Canada for technical assistance with bioinformatic analysis.

\section{Literature Cited}

Bolger, A. M., Lohse, M., and Usadel, B. 2014. Trimmomatic: A flexible trimmer for Illumina sequence data. Bioinformatics 30:2114-2120.

Brodie, B. B. 1996. The identification and distribution of a second pathotype of potato cyst nematodes in the United States. Nematropica 26:246.

Brodie, B. B. 2003. The loss of expression of the $\mathrm{H} 1$ gene in Bt transgenic potatoes. Am. J. Potato Res. 80:135-139.

Brodie, B. B., and Mai, W. F. 1989. Control of the golden nematode in the United States. Annu. Rev. Phytopathol. 27:443-461.
Cingolani, P., Platts, A., Wang, L. L., Coon, M., Nguyen, T., Wang, L., Land, S. J., Lu, X., and Ruden, D. M. 2012. A program for annotating and predicting the effects of single nucleotide polymorphisms, SnpEff: SNPs in the genome of Drosophila melanogaster strain w1118; iso-2; iso-3. Fly (Austin) 6:80-92.

Cook, D. E., and Andersen, E. C. 2017. VCF-kit: Assorted utilities for the variant call format. Bioinformatics 33:1581-1582.

Dandurand, L.-M., Zasada, I. A., Wang, X., Mimee, B., De Jong, W., Novy, R., Whitworth, J., and Kuhl, J. 2019. Current status of potato cyst nematodes in North America. Annu. Rev. Phytopathol. 57:117-133. 
Evans, K., and Brodie, B. B. 1980. The origin and distribution of the golden nematode and its potential in the U.S.A. Am. Potato J. 57:79-89.

Evans, K., and Stone, A. R. 1977. A review of the distribution and biology of the potato cyst nematodes Globodera rostochiensis and G. pallida. Proc. Natl. Acad. Sci. U.S.A. 23: 178-189.

Eves-van den Akker, S., Laetsch, D. R., Thorpe, P., Lilley, C. J., Danchin, E. G. J., Da Rocha, M., Rancurel, C., Holroyd, N. E., Cotton, J. A., Szitenberg, A., Grenier, E., Montarry, J., Mimee, B., Duceppe, M.-O., Boyes, I., Marvin, J. M. C., Jones, L. M., Yusup, H. B., Lafond-Lapalme, J., Esquibet, M., Sabeh, M., Rott, M., Overmars, H., Finkers-Tomczak, A., Smant, G., Koutsovoulos, G., Blok, V., Mantelin, S., Cock, P. J. A., Phillips, W., Henrissat, B., Urwin, P. E., Blaxter, M., and Jones, J. T. 2016. The genome of the yellow potato cyst nematode, Globodera rostochiensis, reveals insights into the basis of parasitism and virulence. Genome Biol. 17:124.

Garrison, E., and Marth, G. 2012. Haplotype-based variant detection from short-read sequencing. arXiv. https://arxiv.org/abs/1207.3907
Kort, J., Ross, H., Rumpenhorst, H. J., and Stone, A. R. 1977. An international scheme for identifying and classifying pathotypes of potato cyst-nematodes Globodera rostochiensis and G. pallida. Nematologica 23:333-339.

Li, H., Handsaker, B., and Wysoker, A. 2009. The sequence alignment/Map format and SAMtools. Bioinformatics 25:2078-2079.

Mai, W. F. 1977. Worldwide distribution of potato-cyst nematodes and their importance in crop production. J. Nematol. 9:30-34.

Mimee, B., Duceppe, M.-O., Véronneau, P.-Y., Lafond-Lapalme, J., Jean, M., Belzile, F., and Bélair, G. 2015. A new method for studying population genetics of cyst nematodes based on Pool-Seq and genomewide allele frequency analysis. Mol. Ecol. Resour. 15:1356-1365.

Plaisted, R. L., Halseth, D. E., Brodie, B. B., Slack, S. A., Sieczka, J. B., Christ, B. J., Paddock, K. M., and Peck, M. W. 1998. Pike: A full season scab and golden nematode resistant chipstock variety. Am. J. Potato Res. 75:117-120.

Whitworth, J. L., Novy, R. G., Zasada, I. A., Wang, X., Dandurand, L.-M., and Kuhl, J. C. 2018. Resistance to potato breeding clones and cultivars to three species of potato cyst nematode. Plant Dis. 102:2120-2128. 